\title{
HIGHLIGHTS
}

\section{RISK FACTORS}

\section{Erectile dysfunction and CVD}

Erectile dysfunction (ED) is inextricably linked with abnormal functioning of the endothelial progenitor cells involved in vascular repair, which has been postulated to be an early mechanism of atherosclerosis. Several studies have identified an association between ED and the presence of cardiovascular risk factors and atherosclerosis. Michael Böhm and coworkers have now added to this field of research by reporting that ED is also directly associated with hard cardiovascular events, including death and myocardial infarction.

The involvement of angiotensin II in the pathogenesis of endothelial dysfunction also led the investigators to speculate that agents that modulate the renin-angiotensin system could have a role in the treatment of ED. Böhm and colleagues, therefore, conducted a substudy of ONTARGET and TRANSCEND to investigate the effects of the angiotensin-convertingenzyme (ACE) inhibitor ramipril and the angiotensin-receptor blocker telmisartan, alone or in combination, on the outcomes of patients with ED.

ONTARGET was a randomized controlled trial comparing ramipril with telmisartan in 25,577 patients with vascular disease or high-risk diabetes. In TRANSCEND, telmisartan was compared with placebo among 1,773 patients with cardiovascular disease or diabetes, with end-organ damage, who were also intolerant to ACE inhibitors.

The ED substudy of these two trials involved 1,549 men who consented to participate. At baseline (enrollment into ONTARGET or TRANSCEND) and after 2 years of follow-up, these patients were asked to complete a questionnaire based on the 5-item short form of the International Index of Erectile Function (IIEF) and the Kölner Evaluation of Erectile Dysfunction scores.

ED at baseline was present in 55\% of participants and was more common among older men with diabetes, hypertension, a prior stroke or transient ischemic attack, or those who had undergone urinary tract surgery. During follow-up (median 53 and 54 months for the ONTARGET and TRANSCEND patient populations, respectively), all-cause mortality was twice as high among individuals with ED than in those without. The rates of cardiovascular death and myocardial infarction were also significantly increased among patients with
ED. There were no differences between the groups treated with telmisartan, ramipril, or both in the prevalence of ED present at baseline or the incidence of new-onset ED. However, treatment effects on blood pressure were evident; among the ONTARGET patients, those receiving telmisartan experienced greater blood pressure reduction than those taking ramipril, with the greatest benefit in the combination-therapy group. In the TRANSCEND population, telmisartan was associated with greater blood pressure lowering effects than was placebo.

"Now we have a true predictor of cardiovascular outcomes, we need to know more about [the] mechanisms" says Dr Böhm. "We have to relate ED to different components of cardiovascular risk." Böhm recommends that "any patient with ED of unknown origin should be presented to a cardiologist in order to evaluate cardiovascular risk and treat previously unknown risk factors."

Alexandra King

Original article Böhm, M. et al. Erectile dysfunction predicts cardiovascular events in high-risk patients receiving telmisartan, ramipril, or both. The ONgoing Telmisartan Alone and in combination with Ramipril Global Endpoint Trial/Telmisartan Randomized AssessmeNt Study in ACE iNtolerant subjects with cardiovascular Disease (ONTARGET/ TRANSCEND) Trials. Circulation 121, 1439-1446 (2010) 